\title{
Economic and Emission Dispatch using Whale Optimization Algorithm (WOA)
}

\author{
Faseela C. K. ${ }^{1}$, H. Vennila ${ }^{2}$ \\ ${ }^{1}$ Departement of Electrical and Electronics Engineering, MES College of Engineering \& Technology, Kerala, India \\ ${ }^{2}$ Departement of Electrical Engineering, Noorul Islam College of Engineering, Nagercoil, TN, India
}

\begin{tabular}{l} 
Article Info \\
\hline Article history: \\
Received Dec 23, 2017 \\
Revised Feb 27, 2018 \\
Accepted Mar 16, 2018 \\
\hline
\end{tabular}

Keyword:

Economic dispatch

Emission cost

Fuel cost

Optimum

Particle swan optimization

Whale optimization algorithm

\begin{abstract}
This paper work present one of the latest meta heuristic optimization approaches named whale optimization algorithm as a new algorithm developed to solve the economic dispatch problem. The execution of the utilized algorithm is analyzed using standard test system of IEEE 30 bus system. The proposed algorithm delivered optimum or near optimum solutions. Fuel cost and emission costs are considered together to get better result for economic dispatch. The analysis shows good convergence property for WOA and provides better results in comparison with PSO. The achieved results in this study using the above-mentioned algorithm have been compared with obtained results using other intelligent methods such as particle swarm Optimization. The overall performance of this algorithm collates with early proven optimization methodology, Particle Swarm Optimization (PSO). The minimum cost for the generation of units is obtained for the standard bus system.
\end{abstract}

Copyright () 2018 Institute of Advanced Engineering and Science. All rights reserved.

\section{Corresponding Author:}

Faseela C. K.,

Departement of Electrical and Electronics Engineering,

MES College of Engineering \& Technology, Kerala, India.

Email: faseelack6@gmail.com

\section{INTRODUCTION}

In today's world, it's always been a concern for an engineer to get a product out at a very optimal cost by minimizing both the product operating cost and raw material input to the production unit. Economic Load Dispatch (ELD) deals with the same situation and it works on operating a coordinated power system such that the lowest operating cost generators are used to the greatest extent and the highest operating cost generator is used to the lowest extent. Economic load dispatch problem is a constrained problem, several principles and strategies are already being developed to solve these problems. ELD has become an important fundamental function in operation and control of the power system.

The demand for electricity is increasing in a large factor in today's life, which makes it highly crucial to run generators at very minimal cost. This is the main factor of an Economic dispatch problem. With the unexceptional production of carbon emissions in thermal power plant, its needed to optimize the emission together with the optimization of cost which acts as two vital parts of Economic dispatch problem. The economic dispatch solution provides the best minimum cost of fuel and emission. This indirectly makes lower cost for electricity and makes electrical utilities more competitive in the market. As the energy cannot be stored, it requires highly efficient estimation scenarios including transmission and distribution systems to make the same work effectively.

Various technologies have been introduced to solve the optimization of Economic Load Dispatch problems. The selection of the optimization algorithm is the important part of the problem involving economic dispatch. The EDP is developed based on real-valued codification. In modern methodology only 
the cost function is evaluated and a global minimum solution is computed, independently of the cost function. The use of digital computers for obtaining loading schedules were investigated and used today.

Many deterministic optimization approaches were proposed to solve the ELD problem, including lambda iteration method [1], gradient method, linear programming [4], non-linear programming, dynamic programming [2] and quadratic programming [14]. But these methods require enormous efforts in terms of computation. Due to complexities of computing, therefore efficient algorithm to find optimal solution like genetic algorithm [16], [18], particle swarm optimization [5], evolutionary programming, artificial bee colony optimization [9], [10], and biogeography based optimization; bacterial foraging and also their variants came into implement. Bio-inspired meta-heuristic algorithms have recently shown the efficiency in dealing with many nonlinear optimizations constrained problems for finding the optimal solution.

Recently a nature based optimization technique whale optimization Algorithm (WOA) is developed based on the flashing behavior of Whales. WOA developed and is used to solve constrained engineering problems. Until now many researches have been carried out to find the closest optimum result in determining the power generation of each generator using WOA and it was inferred that the WOA is more robust and efficient in determining the optimal load scheduling.

\section{PROBLEM FORMULATION}

The generating units are loaded economically such a way to reduce the operating cost. Considering the valve point effect the economic dispatch formulated the objective function as given below

$$
F C_{i}=a_{i}+b_{i} P_{i}+c_{i} P_{i}^{2}
$$

Where

$$
\begin{aligned}
& a_{i}, b_{i}, c_{i} \text { are the fuel cost coefficients of generator }{ }^{i} \\
& \mathrm{P}_{\mathrm{i}} \text { is the power generated by unit } i \text {, MW } \\
& \mathrm{FC}_{\mathrm{i}} \text { is the fuel cost function of unit } i
\end{aligned}
$$

The total fuel cost for the entire system of $\mathrm{N}$ generators can then be calculated as,

$$
F C_{T}=\sum_{i=1}^{N} F C_{i}\left(P_{i}\right)=\sum_{i=1}^{N} a_{i}+b_{i} P_{i}+c_{i} P_{i}^{2}
$$

The new objective function by considering valve point loading along with total fuel cost becomes,

$$
F C_{T}=\sum_{i=1}^{N} a_{i}+b_{i} P_{i}+c_{i} P_{i}^{2}+\left|d_{i} \sin \left(e_{i}\left(P_{i \min }-P_{i}\right)\right)\right|
$$

Where

$$
\begin{aligned}
& a_{i}, b_{i}, c_{i}, d_{i} \text { and } e_{i} \text { are the fuel cost coefficients of generator }{ }^{i} \\
& P_{i} \text { is the power generated by unit }{ }^{i}, \mathrm{MW} \\
& N_{\mathrm{i}} \text { is the number of generating units } \\
& P_{i \text { minis }} \text { the minimum generation limit of unit }{ }^{i}, \mathrm{MW} \\
& F C_{i} \text { is the fuel cost function of unit } i \\
& F C_{T} \text { is the total fuel cost, } \$ / \mathrm{hr} \text {. }
\end{aligned}
$$

In order to minimize the pollutants, emission is consiered along with economic dispatch. The generator can be modelled as having a quadratic relation between the amount of pollutants released and the power generated. 
The mathematical formulation for generator is given by,

$$
E_{i}=\alpha_{i}+\beta_{i} P_{i}+\gamma_{i} P_{i}^{2}
$$

Where

$$
\begin{aligned}
& \alpha_{i}, \beta_{i}, \gamma_{i} \text { are the emission coefficients of generator } i \\
& P_{i} \text { is the power generated by unit } i \text {, MW } \\
& E_{i} \text { is the fuel cost function of unit } i
\end{aligned}
$$

The total emission for the entire system of $\mathrm{N}$ generators can then be calculated as,

$$
E_{T}=\sum_{i=1}^{N} E_{i}\left(P_{i}\right)=\sum_{i=1}^{N} \alpha_{i}+\beta_{i} P_{i}+\gamma_{i} P_{i}^{2}
$$

The new Emission function becomes,

$$
E_{T}=\sum_{i=1}^{N} \alpha_{i}+\beta_{i} P_{i}+\gamma_{i} P_{i}^{2}+\varepsilon_{i} \exp \left(\zeta_{i} P_{i}\right)
$$

Where

$\alpha_{i}, \beta_{i}, \gamma_{i}, \varepsilon_{i}$ and $\zeta_{i}$ are the emission coefficients of generator ${ }^{i}$

$P_{i \text { is the power generated by unit }} i$, MW

$N_{\text {is }}$ the number of generating units

$E_{i \text { is }}$ the emission functieon of unit $i$,

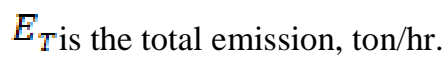

The power balance equation[3] is given by

$$
\sum_{\mathrm{i}=\mathrm{l}}^{\mathrm{N}} \mathbf{P}_{\mathrm{i}=\mathbf{P}_{\mathrm{D}}}
$$

Adding loss factor to the equation.

$$
\sum_{\mathrm{i}=l}^{\mathrm{N}} \mathbf{P}_{\mathrm{i}=} \mathbf{P}_{\mathrm{D}}+\mathbf{P}_{\mathrm{L}}
$$

Power loss PL is calculated as

$$
\mathbf{P}_{L}=\sum_{\mathrm{i}=1}^{N} \quad \sum_{\mathbf{j}=1}^{\mathrm{N}} \mathbf{P}_{\mathbf{i}} \mathbf{B}_{\mathrm{ii}} \mathbf{P}_{\mathbf{i}}+\sum_{\mathrm{i}=1}^{\mathrm{N}} \mathbf{B}_{0 \mathrm{i}} \mathbf{P}_{\mathbf{i}}+\mathbf{B}_{00}
$$

The actual power generation for the generator will be between its maximum and minimum limits which is represented as

$$
\mathbf{P}_{i}^{\min } \leq \mathbf{P i} \leq \mathbf{P}_{\mathbf{1}}^{\max }
$$




\section{WHALE OPTIMIZATION ALGORITHM}

In The mathematical model of whale optimization [6] is detailed in this section.

\subsection{Inspiration}

The hunting method of humpback whales, known as bubble-net-feeding, is used as an inspiration to create this algorithm. This method highlights the intelligence and corporation of humpback whales. The humpback whales hunt in groups (the group size may be as high as a dozen of humpback whales) and the big whale which is the leader finds the group of fishes which is to be hunted. The group goes underneath the water and the lead whale, who is the bubble blower, produces spiral big bubbles towards the surface of the water. These spiral bubbles (shape of ' 9 ') interrupt the fishes to swim through the same and they got struck up within the spiral bubble. Now the group of whales comes out of the water with the open mouth towards the water surface, within the spiral bubble, moving synchronously and hunting all the fishes within the spiral bubble. All the whales forms exactly the same position with respect to the lead whale while hunting. The bubble-net feeding is a unique behavior that can only be observed in humpback whales. In this work, the spiral bubble-net feeding maneuver is mathematically modeled in order to perform optimization.

\subsection{Mathematical model and optimization algorithm}

In this section, the mathematical model of complete behavior of humpback-whale-hunting is done which includes search for small fishes, encircling prey and spiral bubble-net feeding maneuver. The Whale Optimization Algorithm (WOA) is then proposed.

\subsubsection{Encircling prey}

Humpback whales can recognize the location of prey and encircle them. Since the position of the optimal design in the search space is not known a priori, the WOA algorithm assumes that the current best candidate solution is the target prey or is close to the optimum. After the best search agent is defined, the other search agents will hence try to update their positions towards the best search agent. This behavior is represented by the followingequations:

$$
\begin{aligned}
& \vec{D}=\left|\vec{C} \cdot \overrightarrow{X^{*}}(t)-\vec{X}(t)\right| \\
& \vec{X}(t+1)=\overrightarrow{X^{*}}(t)-\vec{A} \cdot \vec{D}
\end{aligned}
$$

where

' $\mathrm{t}$ ' indicates the current iteration,

' $A$ ' and ' $C$ ' are coefficient vectors,

$\mathrm{X} *$ is the position vector of the best solution obtained so far,

$\mathrm{X}$ is the position vector, || is the absolute value, and $\cdot$ is an element-by-element multiplication.

$\mathrm{X} *$ should be updated in each iteration if there is a better solution.

The vectors $\mathrm{A}$ and $\mathrm{C}$ are calculated as follows:

$$
\begin{aligned}
& \vec{A}=2 \vec{a} \cdot \vec{r}-\vec{a} \\
& \vec{C}=2 \cdot \vec{r}
\end{aligned}
$$

where $\vec{a}$ is linearly decreased from 2 to 0 over the course of iterations (in both exploration and exploitation phases) and $\vec{r}^{\prime}$ is a random vector in $[0,1]$.

Figure 1(a) illustrates the rationale behind Equation (12) for a 2D problem. The position $(\mathrm{X}, \mathrm{Y})$ of a search agent can be updated according to the position of the current best record $(\mathrm{X} *, \mathrm{Y} *)$. Different places around the best agent can be achieved with respect to the current position by adjusting the value of $\mathrm{A}$ and $\mathrm{C}$ vectors. The possible updating position of a search agent in 3D space is also depicted in Figure 1(b). It should 
be noted that by defining the random vector $(\vec{r})$ it is possible to reach any position in the search space located between the key-points shown in Figure 2. Therefore, Equation (12) allows any search agent to update its position about the current best solution and simulates encircling the prey.

The same concept can be extended to a search space with $\mathrm{n}$ dimensions, and the search agents will move in hyper-cubes around the best solution obtained so far. As mentioned in the previous section, the humpback whales also attack the prey with the bubble-net strategy. This method is mathematically formulated in the next section.
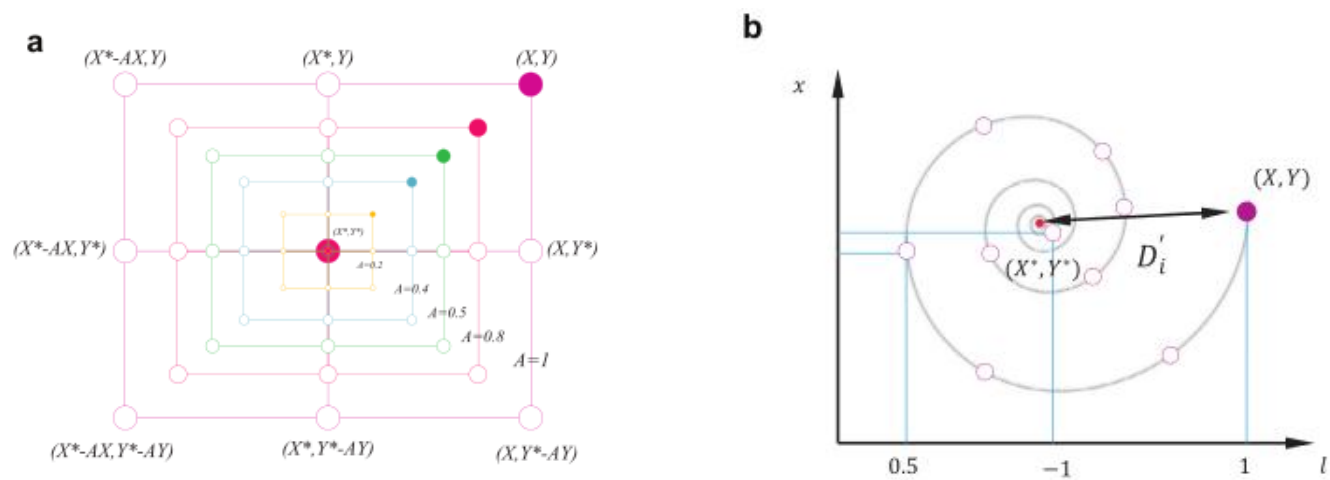

Figure 1. Bubble-net search mechanism implemented in WOA ( $\mathrm{X} *$ is the best solution obtained so far): (a) shrinking encircling mechanism and (b) spiral updating position

\subsubsection{Bubble-net attacking method (exploitation phase)}

To mathematically model the bubble-net behavior of humpback whales, two approaches are designed as follows:

Shrinking encircling mechanism: This behavior is achieved by decreasing the value of $\vec{a}$ in the Equation (13). Note that the fluctuation range of $\vec{A}$ is also decreased by $\vec{a}$. In other words $\vec{A}$ is a random value in the interval $[-\mathrm{a}, \mathrm{a}]$ where $\mathrm{a}$ is decreased from 2 to 0 over the course of iterations. Setting random values for $\vec{A}$ in $[-1,1]$, the new position of a search agent can be defined anywhere in between the original position of the agent and the position of the current best agent. Fig. 3(a) shows the possible positions from $(\mathrm{X}, \mathrm{Y})$ towards $(\mathrm{X} *, \mathrm{Y} *)$ that can be achieved by $0 \leq \mathrm{A} \leq 1$ in a $2 \mathrm{D}$ space.

Spiral updating position: As can be seen in Figure 3(b), this approach first calculates the distance between the whale located at $(\mathrm{X}, \mathrm{Y})$ and prey located at $(\mathrm{X} *, \mathrm{Y} *)$. A spiral equation is then created between the position of whale and prey to mimic the helix-shaped movement of humpback whales as follows:

$$
\vec{X}(t+1)=\overrightarrow{D^{\prime}} \cdot e^{b l} \cdot \cos (2 \pi l)+\vec{X}^{*}(t)
$$

Where $\overrightarrow{D^{\prime}}=\left|\overrightarrow{X^{*}}(t)-\vec{X}(t)\right|$ and indicates the distance of the $\mathrm{i}$ th whale to the prey (best solution obtained so far), $b$ is a constant for defining the shape of the logarithmic spiral, 1 is a random number in $[-1,1]$, and '.' is an element-by-element multiplication. The humpback whales swim around the prey within a shrinking circle and along a spiral-shaped path simultaneously. To model this simultaneous behavior, we assume that there is a probability of $50 \%$ to choose between either the shrinking encircling mechanism or the spiral model to update the position of whales during optimization. The mathematical model is as follows:

$$
\vec{X}(t+1)= \begin{cases}\overrightarrow{X^{*}}(t)-\vec{A} \cdot \vec{D} & \text { if } p<0.5 \\ \overrightarrow{D^{\prime}} \cdot e^{b l} \cdot \cos (2 \pi l)+\overrightarrow{X^{*}}(t) & \text { if } p \geq 0.5\end{cases}
$$

where $\mathrm{p}$ is a random number in $[0,1]$. In addition to the bubble-net method, the humpback whales search for prey randomly. The mathematical model of the search is given in the next section. 


\subsubsection{Search for prey (exploration phase)}

The same approach based on the variation of the A vector can be utilized to search for prey (exploration). In fact, humpback whales search randomly according to the position of each other. Therefore, we use $\mathrm{A}$ with the random values greater than 1 or less than -1 to force search agent to move far away from a reference whale. In contrast to the exploitation phase, we update the position of a search agent in the exploration phase according to a randomly chosen search agent instead of the best search agent found so far. This mechanism and $|\mathrm{A}|>1$ emphasize exploration and allow the WOA algorithm to perform a global search. The mathematical model is as follows:

$$
\begin{gathered}
\vec{D}=\left|\vec{C} \cdot \overrightarrow{X_{\text {rand }}}-\vec{X}\right| \\
\vec{X}(t+1)=\overrightarrow{X_{\text {rand }}}-\vec{A} \cdot \vec{D}
\end{gathered}
$$

\section{WAO FOR ECONOMIC DISPATCH CASE}

Merit order dispatch case is implemented using WOA algorithm. The Economic dispatch case implementation is done on standard IEEE 30 bus system. As it's a standard test system, various parameters had already been recorded. This system was used in many comparable studies in the merit order dispatch.

\subsection{IEEE-30 bus system - results}

This system has 6 generator buses at bus 1,2, 5, 8, 11 and 13. Each of these generators has their own fuel and emission coefficients. These are represented in per unit values to simplify calculations. The base considered is $100 \mathrm{MVA}$. The total demand considered is $2.38 \mathrm{p}$.u. The various generation parameters are tabulated as follows, Generator cost coefficients for IEEE 30 bus system is provided in Table 1 given below.

Table 1. Sample Cost Coefficients

\begin{tabular}{llllllll}
\hline Unit & $\boldsymbol{a}$ & $\boldsymbol{b}$ & $\boldsymbol{c}$ & $\mathrm{e}$ & $\mathrm{F}$ & $\boldsymbol{P}_{\text {i } \min }$ & $\boldsymbol{P}_{\text {i } \max }$ \\
\hline 1 & 10 & 200 & 100 & 15 & 6.283 & 0.05 & 0.5 \\
2 & 10 & 150 & 120 & 10 & 8.976 & 0.05 & 0.6 \\
3 & 20 & 180 & 40 & 10 & 14.784 & 0.05 & 1 \\
4 & 10 & 100 & 60 & 5 & 20.944 & 0.05 & 1.2 \\
5 & 20 & 180 & 40 & 5 & 25.133 & 0.05 & 1 \\
6 & 10 & 150 & 100 & 5 & 18.48 & 0.05 & 0.6 \\
\hline
\end{tabular}

Generator emission coefficients for IEEE-30-bus system is provided in Table 2 given below.

Table 2. Sample Emission Coefficients

\begin{tabular}{llllll}
\hline Unit & $\boldsymbol{\alpha}$ & $\boldsymbol{\beta}$ & $\boldsymbol{\gamma}$ & $\boldsymbol{\varepsilon}$ & $\zeta$ \\
\hline 1 & 4.091 & -5.554 & 6.49 & $2.00 \mathrm{E}-03$ & 2.857 \\
2 & 2.543 & -6.047 & 5.638 & $5.00 \mathrm{E}-04$ & 3.333 \\
3 & 4.258 & -5.094 & 4.586 & $1.00 \mathrm{E}-06$ & 8 \\
4 & 5.326 & -3.35 & 3.38 & $2.00 \mathrm{E}-03$ & 2 \\
5 & 4.258 & -5.094 & 4.586 & $1.00 \mathrm{E}-06$ & 8 \\
6 & 6.131 & -5.555 & 5.151 & $1.00 \mathrm{E}-05$ & 6.667 \\
\hline
\end{tabular}

IEEE-30 bus system simulation and implementation of Economic dispatch using WOA is done using MATLAB program. The following are the results obtained in comparison with PSO algorithm.

The value is found at each step and results are calculated varying the no of iterations and graph is plotted for the same. The best result is obtained at $106^{\text {th }}$ iterations and there is no difference afterwards. 
Table 3. Economic Dispatch results using WOA

\begin{tabular}{lll}
\hline & PSO & WOA \\
\hline$P_{1}$ & 0.099441 & 0.098762 \\
$P_{2}$ & 0.36248 & 0.37468 \\
$P_{3}$ & 0.48349 & 0.47011 \\
$P_{4}$ & 0.87359 & 0.92773 \\
$P_{5}$ & 0.66428 & 0.70221 \\
$P_{6}$ & 0.39004 & 0.38991 \\
Total Fuel Cost & 40752.19 & 40232.26 \\
$\left.F C_{T}\right)$ INR/hr & & \\
Total Emission & 0.213921 & 0.213841 \\
$\left.E_{T}\right)$ ton/hr & & \\
Total Emission Cost & 661.018 & 660.7687 \\
$\left.E C_{T}\right)$ INR/hr & & \\
Total Cost $(T C)$ & 41413.20 & 40893.03 \\
INR/hr & & \\
\hline
\end{tabular}

\section{INFERENCES}

Whale optimization provides excellent results for an economic dispatch problem in terms of cost optimization and easy convergence. On comparison, the cost optimization of WOA is better than PSO by $1.3 \%$. There can be further improvements on the algorithm considering the following factors. Use combinational algorithms - Suggested algorithms are ALO and WOA extracting effective features of both. Include other characteristics of humpback whales on this algorithm. This includes effects of 'double loop' bubbles into this algorithm.

\section{REFERENCES}

[1] Susheel Kumar Dewangan, Achala Jain, Dr. A. P. Huddar, "A Traditional Approach to Solve Economic Load Dispatch Problem Considering the Generator Constraints", IOSR Journal of Electrical and Electronics Engineering (IOSR-JEEE), vol. 10, no. 2 Ver. III (Mar-Apr. 2015), pp. 27-32

[2] D. L.Travers, R. Kaye, "Dynamic dispatch by constructive dynamic programming", IEEE Trans. on Power Systems, vol. 13, no. 1, pp. 72-78, Feb1998.

[3] A. J. Wood and B.F.Wollenberg, Power Generation, Operation and Control, New York: Wiley, (1996).

[4] B. Stott, Hobson, Eric, "Power System Security Control Calculations Using Linear Programming", Part I, IEEE Trans. on Power Apparatus and Systems, vol. PAS-97, no.5, pp. 1713-1720, Sept 1978.

[5] K. S. Kumar, V. Tamilselvan, N. Murali, R. Rajaram, N. S. Sundaram, and T. Jayabarathi, "Economic load dispatch with emission constraints using various PSO algorithms", WSEAS Transactions on Power Systems, vol. 3, no. 9 , pp. 598-607, 2008.

[6] Mirjalili S., A. Lewis, "The Whale Optimization Algorithm”, Advances in Engineering Software , 2016, pp. 51-67

[7] M. Abido, "Environmental/Economic Power Dispatch using Multiobjective Evolutionary Algorithms", IEEE Trans. on Power Systems, vol. 18, no. 4, pp. 1529-1537, Nov 2003

[8] S. Hemamalini, SP. Simon, "Artificial bee colony algorithm for economic load dispatch problem with non-smooth cost functions", Electric Power Components and Systems, vol. 38, no. 7, pp. 786- 803, May (2010).

[9] Vennila .H, Ruban Deva Prakash.T., "A solution for environmental constrained Economisc Dispatch Problems using Honey Bee Algorithm”, International journal of Computer Application, vol. 47, no. 22, pp. 13-17, (2012).

[10] Vennila, H., Ruban Deva Prakash, T., (2012), "Particle Swam Optimisation Technique for solving Economic Dispatch Problems", Elsevier Journal of Procedia Engineering, no. 48, pp. 2009-2021.

[11] Xin-She Yang, S. Deb, Cuckoo Search via Lévy flights, Nature \& Biologically Inspired Computing, NaBIC 2009, World Congress, pp. 210-214.

[12] F. Reid, L. Hasdorff, "Economic Dispatch Using Quadratic Programming”, IEEE Trans. on Power Apparatus and Systems, vol. PAS-92, no. 6, pp.2015- 2023, Nov. 1973.

[13] K. S. Lee and Z. W. Geem, "A New Structural Optimization Method Based on Harmony Search Algorithm Computers and structures", vol. 82, pp. 781-798, 2004.

[14] Gaing, "Particle swarm optimization to solving the economic dispatch considering the generator constraints", IEEE Trans. Power Syst., vol. 18, pp. 1187-1195, Aug. 2003.

[15] Chao-Lung Chiang, "Improved Genetic algorithm for power economic dispatch of units with valve point effects and multiple fuels", IEEE Transactions on power systems, vol 20, no.4, Nov 2005, pp. 1690-1699. 
[16] E. Lin, G. L. Viviani, (1984), "Hierarchical Economic Dispatch for piecewise quadratic cost functions", IEEE Transactions on power apparatus and systems, vol. PAS-103, no.6, June, pp. 1170-1175.

[17] P. Subbaraja, R. Rengaraj, S. Salivahanan, "Enhancement of self adaptive real coded genetic algorithm using Taguchi method for economic dispatch problem", Applied Soft Computing, pp. 1-10.

[18] Girish Kumar, Rameshwar Singh, "Economic Dispatch of Power System Optimization with Power Generation Schedule Using Evolutionary Technique", International Journal of Advanced Research in Electrical Electronics and Instrumentation Engineering, vol. 3, no. 7, July 2014.

[19] Anurag Gupta, K. K. Swarnkar, K. Wadhwani, "Combined Economic Emission Dispatch Problem using Particle Swarm Optimization”, International Journal of Computer Applications (0975-8887), vol. 49, no. 6, July 2012.

[20] Hadi Saadat, Power System Analysis, by Mc Grew Hill. Inc ;1999. 